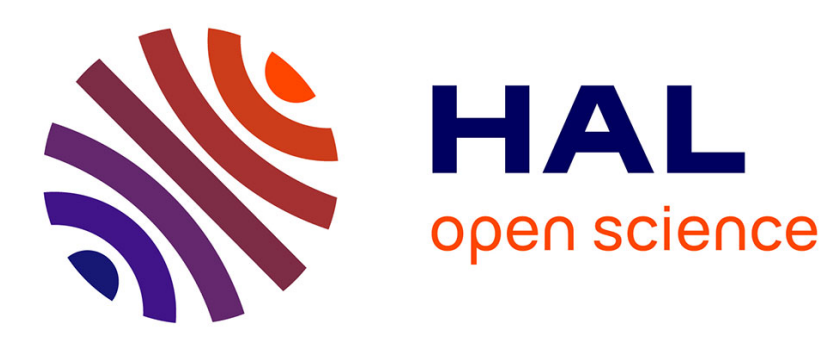

\title{
A relaxation result for an inhomogeneous functional preserving point-like and curve-like singularities in image processing \\ Gilles Aubert, Daniele Graziani
}

\section{- To cite this version:}

Gilles Aubert, Daniele Graziani. A relaxation result for an inhomogeneous functional preserving point-like and curve-like singularities in image processing. 2012. inria-00536206v3

\section{HAL Id: inria-00536206 \\ https://hal.inria.fr/inria-00536206v3}

Submitted on 19 Sep 2012

HAL is a multi-disciplinary open access archive for the deposit and dissemination of scientific research documents, whether they are published or not. The documents may come from teaching and research institutions in France or abroad, or from public or private research centers.
L'archive ouverte pluridisciplinaire HAL, est destinée au dépôt et à la diffusion de documents scientifiques de niveau recherche, publiés ou non, émanant des établissements d'enseignement et de recherche français ou étrangers, des laboratoires publics ou privés. 


\title{
A relaxation result for an inhomogeneous functional preserving point-like and curve-like singularities in image processing
}

\author{
GILLES AUBERT \\ LABORATOIRE J.A. DIEUDONNÉ \\ Université de Nice SOPHIA ANTIPOLIS, Parc Valrose 06108 Nice CEDEX 2, \\ FRANCE \\ email: gaubert@unice.fr \\ DANIELE GRAZIANI ${ }^{1}$ \\ MORPHEME CNRS/INRIA/UNSA SOPHIA ANTIPOLIS \\ Inria, 2004 route des Lucioles BP 9306902 SOPHIA ANTIPOLIS CEDEX, FRANCE \\ email: daniele.graziani@inria.fr
}

\begin{abstract}
In the present paper we address a relaxation theorem for a new integral functional of the calculus of variations defined on the space of functions in $W_{\text {loc }}^{1, p}$ whose gradient is an $L^{p}$-vector field with distributional divergence given by a Radon measure. The result holds for integrand of type $f(x, \Delta u)$ without any coerciveness condition, with respect to the second variable, and $C^{1}$-continuity assumptions with respect to the spatial variable $x$.
\end{abstract}

AMS subject Classification: 49J45 49M20 49Q20

Keywords: $W_{\text {loc }}^{1, p}$-lower semicontinuity, divergence measure fields, Leibniz rule, p-capacity, convexity, relaxation, image processing.

\section{INTRODUCTION}

In a recent paper (see [2]) the authors have introduced a new functional of calculus of variations to preserve point-like and curve-like singularities in biological images corrupted by noise. The energy, they deal with, was the following

$$
\begin{aligned}
\mathcal{F}\left(u, \Omega^{\prime}\right): & =\int_{\Omega^{\prime}} f(\Delta u) d x+\int_{\Omega^{\prime}} f^{\infty}\left(\frac{d \mu^{a}}{d\left|\mu^{a}\right|}\right) d\left|\mu^{a}\right|+\int_{\Omega^{\prime}} f^{\infty}\left(\frac{d \mu^{0}}{d\left|\mu^{0}\right|}\right) d\left|\mu^{0}\right|+\int_{\Omega^{\prime}} g(\nabla u) d x \\
& +\int_{\Omega^{\prime}}\left|u-u_{0}\right|^{2} d x
\end{aligned}
$$

\footnotetext{
${ }^{1}$ Corresponding author.
} 
where $\Omega^{\prime}$ is an arbitrary open set such that $\Omega^{\prime} \subset \subset \Omega \subset \mathbb{R}^{N} . N \in\{2,3\}, u \in \Delta \mathcal{M}_{\text {loc }}^{p}(\Omega)$ with $\frac{2 N}{N+2} \leq p<\frac{N}{N-1}$. $\Delta \mathcal{M}^{p}(\Omega)$ is the space of $W_{\text {loc }}^{1, p}$-functions whose gradient is an $L^{p}$-vector field with distributional divergence given by a Radon measure; the measures $\mu^{a}, \mu^{0}$ are given by the $p$-capacitary decomposition applied to the singular part of the divergence measure of $\nabla u$, that is

$$
\operatorname{Div} \nabla u=\Delta u d x+(\Delta u)^{s}=\Delta u d x+\mu^{a}+\mu^{0}
$$

$\frac{d \mu^{a}}{d\left|\mu^{a}\right|}, \frac{d \mu^{0}}{d\left|\mu^{0}\right|}$ are the Radon-Nikodym derivatives of the measures $\mu^{a}$ and $\mu^{0}$ with respect to their total variation. The restriction $p<\frac{N}{N-1}$ is needed to allow singularities on curves and points (see $[3,14]$ on this issue). The integrands $f, g$ are convex functions and $f^{\infty}$ is the recession function (see [2] for a precise definition of all these quantities and references on $p$-capacity). Finally $u_{0}$ is a given data and here the restriction $p \geq \frac{2 N}{N+2}$ is assumed to give sense to the discrepancy term $\int_{\Omega^{\prime}}\left|u-u_{0}\right|^{2} d x$. In [2], under suitable growth assumptions on the integrands $f$ and $g$, an existence result was proven. It was also shown that functional (1.1) coincides with the lower semicontinuous envelope, with respect to $W_{\text {loc }}^{1, p}(\Omega)$-weak convergence of the following functional

$$
F(u, \Omega):= \begin{cases}\int_{\Omega} f(\Delta u) d x+\int_{\Omega} g(\nabla u) d x+\int_{\Omega}\left|u-u_{0}\right|^{2} d x & \text { on } C^{2}(\Omega), \\ +\infty & \text { on } \Delta \mathcal{M}_{\mathrm{loc}}^{p}(\Omega) \backslash C^{2}(\Omega) .\end{cases}
$$

For the applications it can be crucial to allow a dependence with respect to the spatial variable $x$ in the integrand $f$. Indeed in the damaged image reconstruction problem one might like to emphasize the singularities contained in a given region of $\Omega$ by giving appropriate values to the integrand. Moreover such $x$-dependence, in most cases, does not satisfy strong regularity property, as strong differentiability for instance. Typical examples are integrand of type $f(x, \xi)=a(|x|) f(\xi)$. Therefore it makes sense, even from an experimental point of view, to consider integrand of type $f(x, \Delta u)$. However in this work we have limited ourselves to a pure theoretical analysis. We refer to [2] for numerical applications in the homogeneous case.

In view of integral representation formula, it is natural to investigate lower semicontinuity property when such an $x$-dependence is allowed. Indeed the lower semicontinuity of functional $\mathcal{F}$ corresponds to obtain the so called "lim inf" inequality (see subsection 2.6) for the relaxed functional of $F$. Here we disregard the data term $\int_{\Omega}\left|u-u_{0}\right|^{2}$, since it is a continuous perturbation that does not affect the integral representation of the relaxed functional. Moreover, since decomposition (1.2) plays no role in the proof of the relaxation result, we split the Radon measure Div $\nabla u$ by using the canonical 
Radon-Nikodym decomposition:

$$
\operatorname{Div} \nabla u=\Delta u d x+(\Delta u)^{s} .
$$

Therefore in this paper we first study the lower semicontinuity, with respect to the $W_{\text {loc }}^{1, p}(\Omega)$-weak convergence, of the following inhomogeneous version of functional (1.1):

$$
\mathcal{F}\left(u, \Omega^{\prime}\right):=\int_{\Omega^{\prime}} f(x, \Delta u) d x+\int_{\Omega^{\prime}} f^{\infty}\left(x, \frac{d(\Delta u)^{s}}{d\left|(\Delta u)^{s}\right|}\right) d\left|(\Delta u)^{s}\right|+\int_{\Omega^{\prime}} g(\nabla u) d x .
$$

with $\Omega^{\prime} \subset \subset \Omega$. In order to prove $W_{\text {loc }}^{1, p}(\Omega)$-weak lower semicontinuity result we use a successful technique, developed in these last years, to address the $L^{1}$-lower semicontinuity of integral functionals (even if the integrand depends on the variable $u$ ) defined on the space $B V(\Omega)$. It permits to prove lower semicontinuity theorems by dropping the coerciveness assumptions and under weak differentiability requirements on the integrand $f(x, u, \nabla u)$. The main tools of this approach are a chain rule formula and an approximation result for families of convex functions depending on a parameter due to E.De Giorgi (see Theorem 2.2). There is by now a vast literature on this topic. Without claiming of being exhaustive we refer to $[13,16]$ and references therein for an overview on this subject.

Very roughly speaking, by De Giorgi's Theorem, one can write functional $\mathcal{F}$ as a supremum of affine functionals involving the scalar product between certain coefficients (the so-called De Giorgi's coefficients) and the derivatives of $u$. Then if $\left\{u_{n}\right\} \subset \Delta \mathcal{M}_{\text {loc }}^{p}(\Omega)$ is a sequence which converges to a function $u \in \Delta \mathcal{M}_{\text {loc }}^{p}(\Omega)$, one can recover the convergence of the derivatives of $u_{n}$, by switching the derivatives to suitable test functions and so proving the continuity of those affine functionals and therefore the lower semicontinuity of $\mathcal{F}$.

In this paper we adapt this strategy to our different variational framework in order to obtain $W_{\text {loc }}^{1, p}$-weak lower semicontinuity under weak regularity conditions on the integrand with respect to the spatial variable $x$. In particular a new Leibniz rule for the product between a proper scalar function $b$ and $u \in \Delta \mathcal{M}^{p}(\Omega)$ is established. Besides in order to deal with the right duality involving the $W_{\text {loc }}^{1, p}(\Omega)$-weak convergence, we assume that the integrand $f$ belongs to $W^{1, p^{\prime}}(\Omega)$ with $\nabla_{x} f \in L_{\text {loc }}^{p^{\prime}}(\Omega \times \mathbb{R}), \frac{1}{p}+\frac{1}{p^{\prime}}=1$ (see assumption (4.1)).

The last part of the paper is devoted to provide the so called "limsup" inequality for the relaxed functional of $F$, which, combined with the "liminf" inequality, gives the integral representation $\bar{F}=\mathcal{F}$ (see subsection 2.6 and Theorem 5.1). The proof is based on an approximation result for lower semicontinuous functions contained in 
[12]. This result, under a suitable uniform lower semicontinuity condition with respect to the spatial variable (see Theorem 2.3), permits to write a convex integrand $f$ as a supremum of functions which are split as a product of a function depending only on the spatial variable times a function only depending on the second variable. Then in order to prove "limsup" inequality we can adapt the technique used in [2] for the isotropic functional (1.1). Moreover to attain the upper bound we need, as in the case of integral functional defined on $B V$-space, to require a linear growth from above on the integrand $f$.

The paper is organized as follows. Section 2 is devoted to notations, preliminary definitions and results. In Section 3 we address the new Leibniz rule formula. In section 4 we prove the lower semicontinuity result. Finally in section 5 we provide the integral representation formula.

\section{DEFINITION AND MAIN PROPERTIES}

2.1. Distributional divergence and classical spaces. In this subsection we recall the definition of the distributional space $L^{p, q}(\operatorname{Div} ; \Omega)$ and $\mathcal{D} \mathcal{M}^{p}(\Omega), 1 \leq p, q \leq+\infty$, (see $[1,6,7]$ ). In all the paper $\Omega \subset \mathbb{R}^{N}$ is an open bounded set with Lipschitz boundary. $N \in\{2,3\}$ is the space dimension. $\mathcal{L}^{n}$ and $d x$ will denote the Lebesgue measure on $\mathbb{R}^{N}$. The bracket $\langle\cdot, \cdot\rangle$ stands for the duality product in some distributional space.

Definition 2.1. We say that $U \in L^{p, q}(\operatorname{Div} ; \Omega)$ if $U \in L^{p}\left(\Omega ; \mathbb{R}^{N}\right)$ and if its distributional divergence $\operatorname{Div} U \in L^{q}(\Omega)$. If $p=q$ the space $L^{p, q}(\operatorname{Div} ; \Omega)$ will be denoted by $L^{p}(\operatorname{Div} ; \Omega)$.

Definition 2.2. For $U \in L^{p}\left(\Omega ; \mathbb{R}^{N}\right), 1 \leq p \leq+\infty$, set

$$
|\operatorname{Div} U|(\Omega):=\sup \left\{\langle U, \nabla \varphi\rangle: \varphi \in C_{0}^{\infty}(\Omega),|\varphi| \leq 1\right\} .
$$

We say that $U$ is an $L^{p}$-Divergence measure field, i.e. $U \in \mathcal{D} \mathcal{M}^{p}(\Omega)$, if

$$
\|U\|_{\mathcal{D M}^{p}(\Omega)}:=\|U\|_{L^{p}\left(\Omega ; \mathbb{R}^{N}\right)}+|\operatorname{Div} U|(\Omega)<+\infty .
$$

We say that $U \in \mathcal{D} \mathcal{M}_{\mathrm{loc}}^{p}(\Omega)$ if $U \in \mathcal{D} \mathcal{M}^{p}\left(\Omega^{\prime}\right)$ for every $\Omega^{\prime} \subset \subset \Omega$.

We recall that $U \in L^{p}\left(\Omega ; \mathbb{R}^{N}\right)$ belongs to $\mathcal{D} \mathcal{M}^{p}(\Omega)$ if and only if there exists a Radon measure denoted by $\operatorname{Div} U$ such that

$$
\langle U, \nabla \varphi\rangle=-\int_{\Omega} \operatorname{DivU} \varphi \quad \forall \varphi \in C_{0}^{\infty}(\Omega),
$$

and the total variation of the measure $\operatorname{Div} U$ is given by $|\operatorname{Div} U|(\Omega)$

Let us recall the following classical result (see [7] Proposition 3.1). 
Theorem 2.1. Let $\left\{U_{h}\right\}_{h} \subset \mathcal{D M}^{p}(\Omega)$ be such that

$$
U_{h} \rightarrow U \quad \text { in } L^{p}\left(\Omega ; \mathbb{R}^{N}\right) \text {, as } h \rightarrow+\infty \text { for } 1 \leq p<+\infty .
$$

Then

$$
\|U\|_{L^{p}\left(\Omega ; \mathbb{R}^{N}\right)} \leq \liminf _{h \rightarrow+\infty}\left\|U_{h}\right\|_{L^{p}\left(\Omega ; \mathbb{R}^{N}\right)}, \quad|\operatorname{Div} U|(\Omega) \leq \liminf _{h \rightarrow+\infty}\left|\operatorname{Div} U_{h}\right|(\Omega) .
$$

Finally we define the following space

$$
\Delta \mathcal{M}_{\mathrm{loc}}^{p}(\Omega):=\left\{u \in W_{\mathrm{loc}}^{1, p}(\Omega), \nabla u \in \mathcal{D} \mathcal{M}_{\mathrm{loc}}^{p}(\Omega)\right\} .
$$

For $u \in \Delta \mathcal{M}_{\mathrm{loc}}^{p}(\Omega)$ the measure $\operatorname{Div} \nabla u$ is also denoted with $\Delta u$.

2.2. Preliminary Lemmas. We recall a classical result due to E.De Giorgi, G.Buttazzo and G.Dal Maso (see [10]).

Lemma 2.1. Let $\mu$ be a positive Radon measure on an open set $\Omega \subset \mathbb{R}^{N}$. Consider a sequence $\left\{u_{l}\right\}$ of Borel measurable functions such that for every $l \in \mathbb{N}, u_{l}: \Omega \rightarrow[0, \infty]$. Then

$$
\int_{\Omega} \sup _{l} u_{l} d \mu=\sup _{\left\{A_{k}\right\}_{k \in \mathbb{N}}} \sup _{l \in \mathbb{N}}\left\{\sum_{k=1}^{l} \int_{A_{k}} u_{k} d \mu\right\},
$$

where $A_{k} \subset \Omega$ are open and pairwise disjoint sets with compact closure in $\Omega$.

2.3. Convex Functions. We briefly recall the classical approximation theorem due to E.De Giorgi (see [9]). We state this result in the particular form we will use in the sequel. For more general statement we refer to [9].

Theorem 2.2. Let $f: \Omega \times \mathbb{R} \rightarrow[0,+\infty),(x, t) \mapsto f(x, t)$, be a Borel function convex with respect to $t$ for all $x \in \Omega$. There exists a sequence $\left\{\xi_{l}\right\} \subset C_{0}^{\infty}(\mathbb{R})$ with $\xi_{l} \geq 0$ and $\int_{\mathbb{R}} \xi_{l}(t) d t=1$, such that

$$
f(x, t)=\sup _{l \in \mathbb{N}}\left(\alpha_{l}(x)+\beta_{l}(x) t\right)^{+},
$$

where

$$
\begin{aligned}
\alpha_{l}(x) & :=\int_{\mathbb{R}} f(x, t)\left(2 \xi_{l}(t)+\xi_{l}^{\prime}(t) t\right) d t \\
b_{l}(x) & :=-\int_{\mathbb{R}} f(x, t) \xi_{l}^{\prime}(t) d t .
\end{aligned}
$$

It is worth noticing that the coefficients $\alpha_{l}$ and $\beta_{l}$ explicitly depend on the function $f$. The explicit formulas permit to deduce regularity properties of the coefficients $\alpha_{l}$ and $\beta_{l}$ from proper hypotheses satisfied by $f$. 
We conclude this section with another approximation result for convex function contained in [12]. Also in this case we state the result in a simpler case and we refer the reader to Lemma 8 of [12] for a more general statement.

Theorem 2.3. Let $f: \Omega \times \mathbb{R} \rightarrow[0,+\infty)$ be a lower semicontinuous function in $(x, t)$ such that $f(x, \cdot)$ is convex for all $x \in \Omega$. Assume in addition that for all $\epsilon>0$ and for all $x_{0} \in \Omega$ there exists $\delta>0$ such that

$$
f\left(x_{0}, t\right) \leq f(x, t)+\epsilon(1+f(x, t)) \quad \text { for all }(x, t) \in \Omega \times \mathbb{R} \text { such that }\left|x-x_{0}\right|<\delta .
$$

Then there exist two sequences $\left\{a_{k}\right\} \subset C_{0}^{\infty}(\Omega)$ and $\left\{\psi_{k}\right\} \subset C_{0}^{\infty}(\Omega)$ such that, for all $k \in \mathbb{N}, 0 \leq a_{k} \leq 1, \psi_{k}$ is a convex function satisfying

$$
0 \leq \psi_{k}(t) \leq \Lambda_{k}(1+|t|)
$$

for some $\Lambda_{k} \geq 0$, and

$$
f(x, t)=\sup _{k \in \mathbb{N}} a_{k}(x) \psi_{k}(t) \quad \text { for all }(x, t) \in \Omega \times \mathbb{R} .
$$

2.4. Functionals and their properties. We denote by $\mathcal{A}(\Omega)$, the family of all open bounded subsets $\Omega^{\prime} \subset \subset \Omega$.

Let $f: \Omega \times \mathbb{R} \rightarrow[0,+\infty)$ be a Borel function continuous with respect to the spatial variable and convex in the second variable. We shall consider the following functionals defined for $u \in \Delta \mathcal{M}_{\mathrm{loc}}^{p}(\Omega)$ and $A \in \mathcal{A}(\Omega)$ :

$$
\begin{aligned}
& F(u, \Omega):= \begin{cases}\int_{\Omega} f(x, \Delta u) d x+\int_{\Omega} g(\nabla u) d x & \text { on } C^{2}(\Omega), \\
+\infty & \text { on } \Delta \mathcal{M}_{\mathrm{loc}}^{p}(\Omega) \backslash C^{2}(\Omega) ;\end{cases} \\
& \mathcal{F}\left(u, \Omega^{\prime}\right):=\int_{\Omega^{\prime}} f(x, \Delta u) d x+\int_{\Omega^{\prime}} f^{\infty}\left(x, \frac{d(\Delta u)^{s}}{d\left|(\Delta u)^{s}\right|}\right) d\left|(\Delta u)^{s}\right|+\int_{\Omega^{\prime}} g(\nabla u) d x,
\end{aligned}
$$

where $f^{\infty}$ is the recession function given by $\lim _{t \rightarrow+\infty} \frac{f(x, t \xi)}{t}$, with $\xi \in \mathbb{R}$ and the measure $(\Delta u)^{s}$ are given by classical decomposition applied to the Radon measure $\Delta u \cdot \frac{d(\Delta u)^{s}}{d\left|(\Delta u)^{s}\right|}$ is the Radon-Nikodym derivatives of the singular measure $(\Delta u)^{s}$ with respect to its total variation. We recall that since $f$ is convex $f^{\infty}$ is a well defined Borel function convex in the second variable.

Remark 2.1. The continuity assumption with respect to $x$ is is needed in order to identify $f$ with its continuous representative, otherwise the term $\int_{\Omega^{\prime}} f^{\infty}\left(x, \frac{d(\Delta u)^{s}}{d\left|(\Delta u)^{s}\right|}\right) d\left|(\Delta u)^{s}\right|$ in functional (2.7) may differ if one considers different continuous representative. 
2.5. Relaxation. Let $F$ be the functional defined in (2.6). For every $u \in \Delta \mathcal{M}_{\text {loc }}^{p}(\Omega)$, we define the lower semicontinuous envelope or relaxed functional with respect to the $W_{\text {loc }}^{1, p}$-weak convergence of $F$ given by:

$$
\bar{F}\left(u, \Omega^{\prime}\right):=\inf _{u_{h} \subset C^{2}\left(\Omega^{\prime}\right)}\left\{\liminf _{h \rightarrow+\infty} F\left(u_{h}, \Omega^{\prime}\right) \quad u_{h} \rightarrow u\right\},
$$

where $\Omega^{\prime} \subset \subset \Omega$ is an arbitrary open set.

For the convenience we recall here that a sequence $\left\{u_{h}\right\} \subseteq W_{\text {loc }}^{1, p}(\Omega) W_{\text {loc }}^{1, p}$-weakly converges to $u \in W_{\text {loc }}^{1, p}\left(\Omega^{\prime}\right)$ if and only if for all $\varphi \in W^{1,-p^{\prime}}\left(\Omega^{\prime}\right)$ and $\Omega^{\prime} \subset \subset \Omega$ we have

$$
\left\langle u_{h}, \varphi\right\rangle_{W^{1, p}\left(\Omega^{\prime}\right) \times W^{1,-p^{\prime}}\left(\Omega^{\prime}\right)} \rightarrow\langle u, \varphi\rangle_{W^{1, p}\left(\Omega^{\prime}\right) \times W^{1,-p^{\prime}}\left(\Omega^{\prime}\right)} .
$$

If $L: \Delta \mathcal{M}_{\mathrm{loc}}^{p}(\Omega) \rightarrow \mathbb{R}$ is a continuous operator with respect to the $W_{\text {loc }}^{1, p}(\Omega)$-weak convergence we have:

$$
\overline{(F+L)}=\bar{F}+L \text {. }
$$

For general properties of the relaxation we refer to $[4,5]$.

\section{LeibNiz Rule}

For our purpose the Leibniz rule here below plays a crucial role.

Lemma 3.1. Let $1<p<\frac{N}{N-1}$.

Let $b$ be a scalar function belonging to $W^{1, p^{\prime}}(\Omega) \cap C^{0}(\Omega)$, with $\frac{1}{p}+\frac{1}{p^{\prime}}=1$. Then for every $u \in \Delta \mathcal{M}_{\mathrm{loc}}^{p}(\Omega)$ and for every $\phi \in C_{0}^{\infty}(\Omega)$, the following formula holds:

$$
\int b(x) \phi(x) d(\operatorname{Div} \nabla u)=-\int_{\Omega} \nabla u(x) \cdot \nabla(b(x) \phi(x)) d x .
$$

Remark 3.1. The continuity assumption is needed in order to identify $b$ with its continuous representative, otherwise the previous formula is false.

The proof is based on approximation argument.

Proof. Let $\left\{\rho_{\epsilon}\right\}$ be a standard sequence of mollifiers. We set $b_{\epsilon}=b * \rho_{\epsilon}$. Since the support of $b$ is strictly contained in $\Omega, b_{\epsilon}$ is well defined in $\Omega$. Then, by definition of distributional divergence and taking into account that $b_{\epsilon} \phi \in C_{0}^{\infty}(\Omega)$, we have

$$
\int b_{\epsilon}(x) \phi(x) d(\operatorname{Div} \nabla u)=-\int_{\Omega} \nabla u(x) \cdot \nabla\left(b_{\epsilon}(x) \phi(x)\right) d x .
$$

Now we have $b_{\epsilon} \phi \rightarrow b \phi$ uniformly in $\Omega$ and $\nabla\left(b_{\epsilon} \phi\right) \rightarrow \nabla(b \phi)$ in $L^{p^{\prime}}\left(\Omega ; \mathbb{R}^{N}\right)$. Then we achieve the thesis by taking the limit as $\epsilon \rightarrow 0$ in (3.1). 


\section{LOWER SEMICONTINUITY}

In this section the lower semicontinuity result for functional (2.7) is addressed. The result is obtained by means of Theorem 2.2 and Lemma 3.1.

Let $\Omega \subset \mathbb{R}^{N}$ be a bounded open set. Let $f: \Omega \times \mathbb{R} \rightarrow[0,+\infty)$ be a Borel function, convex in the second variable, which satisfies the following condition:

$$
\left\{\begin{array}{l}
f(\cdot, t) \in W^{1, p^{\prime}}(\Omega) \cap C^{0}(\Omega) \text { with: } \\
\nabla_{x} f \in L_{\mathrm{loc}}^{p^{\prime}}(\Omega \times \mathbb{R}) .
\end{array}\right.
$$

Theorem 4.1. Let $f: \Omega \times \mathbb{R} \rightarrow[0,+\infty)$ be a Borel function convex in the second variable satisfying (4.1). Let $g: \mathbb{R}^{N} \rightarrow[0,+\infty)$ be a convex function. Then functional (2.7) is lower semicontinuous on $\Delta \mathcal{M}_{\mathrm{loc}}^{p}(\Omega)$ with respect to the $W_{\text {loc }}^{1, p}$-weak convergence.

\section{Proof.}

We have to show that for an arbitrary open set $A \subset \subset \Omega$ and for every sequence $\left\{u_{h}\right\} \subseteq$ $W_{\text {loc }}^{1, p}(A) W_{\text {loc }}^{1, p}$-weakly convergent to $u \in W_{\text {loc }}^{1, p}(A)$, th following equality holds:

$$
\liminf _{h \rightarrow+\infty} \mathcal{F}\left(u_{h}, A\right) \geq \mathcal{F}(u, A) .
$$

If $A \subset \subset \Omega$ is an arbitrary open set, we set

$$
\mathcal{G}(u, A):=\int_{A} f(x, \Delta u) d x+\int_{A} f^{\infty}\left(x, \frac{d(\Delta u)^{s}}{d\left|(\Delta u)^{s}\right|}\right) d\left|(\Delta u)^{s}\right| .
$$

By Theorem $(2.2)$ there exists a sequence $\left\{\xi_{l}\right\} \subset C_{0}^{\infty}(\mathbb{R})$ with $\xi_{l} \geq 0$ and $\int_{\mathbb{R}} \xi_{l} d x=1$ such that for any $(x, t) \in \mathbb{R}$ we have

$$
f(x, t)=\sup _{l \in \mathbb{N}}\left(\alpha_{l}(x)+\beta_{l}(x) t\right)^{+}
$$

and

$$
f^{\infty}(x, t)=\sup _{l \in \mathbb{N}}\left(\beta_{l}(x) t\right)^{+},
$$

where, recalling (2.3) and (2.4)

$$
\begin{aligned}
\alpha_{l}(x) & =\int_{\mathbb{R}} f(x, t)\left(2 \xi_{l}(t)+\xi_{l}^{\prime}(t)\right) d t \\
\beta_{l}(x) & =-\int_{\mathbb{R}} f(x, t) \xi_{l}^{\prime}(t) d t .
\end{aligned}
$$

Then for every $u \in \Delta \mathcal{M}_{\text {loc }}^{p}(\Omega)$, we have

$$
\mathcal{G}(u, A)=\int_{A} \sup _{l \in \mathbb{N}}\left(\alpha_{l}(x)+\beta_{l}(x) \Delta u(x)\right)^{+} d x+\int_{A} \sup _{l \in \mathbb{N}}\left(\beta_{l}(x) \frac{d(\Delta u)^{s}}{d\left|(\Delta u)^{s}\right|}\right)^{+} d\left|(\Delta u)^{s}\right| .
$$

Since the measures $d x$ and $\left|(\Delta u)^{s}\right|$ are mutually singular we have:

$$
\mathcal{G}(u, A)=\int_{A} \sup _{l \in \mathbb{N}}\left(\left(\alpha_{l}(x)+\beta_{l}(x) \frac{\Delta u(x)}{|\Delta u(x)|}\right)^{+}+\left(\beta_{l}(x) \frac{d(\Delta u)^{s}}{d\left|(\Delta u)^{s}\right|}(x)\right)^{+}\right) d|\operatorname{Div} u| .
$$


Hence, by applying Lemma 2.1, with $\mu=|\operatorname{Div} \nabla u|(A)$, we obtain

$$
\begin{aligned}
\mathcal{G}(u, A) & =\sup _{\left\{A_{k}\right\}_{k \in \mathbb{N}}} \sup _{l \in \mathbb{N}} \sum_{k=1}^{l}\left(\int_{A_{k}}\left(\alpha_{k}(x)+\beta_{k}(x) \Delta u(x)\right)^{+} d x\right. \\
& \left.+\int_{A_{k}}\left(\beta_{k}(x) \frac{d(\Delta u)^{s}}{d\left|(\Delta u)^{s}\right|}(x)\right)^{+} d\left|(\Delta u)^{s}\right|\right)
\end{aligned}
$$

where $A_{k} \subset A$ are open and pairwise disjoint.

Let now for all $k, \eta_{k}$ be a test function in $C_{0}^{\infty}\left(A_{k}\right)$ with $0 \leq \eta_{k} \leq 1$. As $d x$ and $\left|(\Delta u)^{s}\right|$ are mutually singular measures

$$
\begin{aligned}
\mathcal{G}(u, A) & =\sup _{\left\{A_{k}\right\}_{k \in \mathbb{N}}} \sup _{l \in \mathbb{N}} \sum_{k=1}^{l}\left(\operatorname { s u p } _ { 0 \leq \eta _ { k } \leq 1 } \left(\int_{A_{k}} \alpha_{k}(x) \eta_{k}(x) d x+\int_{A_{k}} \beta_{k}(x) \eta_{k}(x) \Delta u(x) d x\right.\right. \\
& \left.\left.+\int_{A_{k}} \beta_{k}(x) \frac{d(\Delta u)^{s}}{d\left|(\Delta u)^{s}\right|}(x) \eta_{k}(x) d\left|(\Delta u)^{s}\right|\right)\right) .
\end{aligned}
$$

Let us define

$H\left(u, A_{k}\right):=\int_{A_{k}} \alpha_{k}(x) \eta_{k}(x)+\int_{A_{k}} \beta_{k}(x) \Delta u(x) \eta_{k}(x) d x+\int_{A_{k}} \beta_{k}(x) \frac{d(\Delta u)^{s}}{d\left|(\Delta u)^{s}\right|}(x) \eta_{k}(x) d \mid(\Delta u)^{s}$

We are going to prove the continuity of $H$ with respect to the $W_{\text {loc }}^{1, p}(\Omega)$-weak convergence for every $k$ fixed, by applying Lemma 3.1 with $\beta_{k}$ and $u$. Therefore, we need to check first that $\beta_{k}(x)$ satisfies the hypotheses of Lemma 3.1. For every test $\Phi \in C_{0}^{\infty}\left(\Omega ; \mathbb{R}^{N}\right)$ we have

$$
\left\langle\nabla \beta_{k}(x), \Phi(x)\right\rangle_{\mathcal{D}^{\prime}(\Omega)}=-\int_{\Omega} \beta_{k}(x) \operatorname{Div} \Phi(x) d x=\int_{\Omega}\left(\int_{\mathbb{R}} f(x, t) \xi_{k}^{\prime}(t) d t\right) \operatorname{Div} \Phi(x) d x .
$$

Since $\nabla_{x} f \in L^{p^{\prime}}(\Omega \times \mathbb{R})$ we can apply Fubini's theorem to get that

$$
\begin{aligned}
\left\langle\nabla \beta_{k}(x), \Phi(x)\right\rangle_{\mathcal{D}^{\prime}(\Omega)} & =\int_{\mathbb{R}} \xi_{k}^{\prime}(t) d t \int_{\Omega} f(x, t) \operatorname{Div} \Phi(x) d x \\
& =-\int_{\mathbb{R}} \xi_{k}^{\prime}(t) d t \int_{\Omega} \nabla_{x} f(x, t) \cdot \Phi(x) d x .
\end{aligned}
$$

Hence,

we conclude that

$$
\left\langle\nabla \beta_{k}(x), \Phi(x)\right\rangle_{\mathcal{D}^{\prime}(\Omega)}=-\int_{\Omega}\left(\int_{\mathbb{R}} \nabla_{x} f(x, t) \xi_{k}^{\prime}(t) d t\right) \cdot \Phi(x) d x,
$$

and therefore we have the identification:

$$
\nabla \beta_{k}=-\int_{\mathbb{R}} \nabla_{x} f(x, t) \xi_{k}^{\prime}(t) d t
$$

Then, since $\xi_{k} \subset C_{0}^{\infty}(\mathbb{R})$ and $\nabla_{x} f \in L_{\text {loc }}^{p^{\prime}}(\Omega \times \mathbb{R})$ we infer $\beta_{k} \in W^{1, p^{\prime}}(\Omega)$. Finally it is not difficult that, since $f$ is continuous, $\beta_{k}$ is continuous too. 
We are now position of proving the continuity of $H$. Let $\left\{u_{h}\right\} \subset \Delta M_{\text {loc }}^{p}(\Omega)$ be a sequence $W_{\text {loc }}^{1, p}$-weakly converging to $u \in \Delta \mathcal{M}_{\text {loc }}^{p}(\Omega)$. By applying Lemma 3.1

$$
\begin{aligned}
\lim _{h \rightarrow+\infty} H\left(u_{h}, A_{k}\right)= & \int_{A_{k}} \alpha_{k}(x) \eta_{k}(x) d x+\lim _{h \rightarrow+\infty}-\int_{A_{k}}\left(\beta_{k}(x) \nabla u_{h}(x)\right) \cdot \nabla \eta_{k}(x) d x \\
& -\int_{A_{k}} \nabla \beta_{k}(x) \cdot \nabla u_{h}(x) \eta_{k}(x) d x \\
= & \int_{A_{k}} \alpha_{k}(x) \eta_{k}(x) d x+\int_{A_{K}}\left(\beta_{k}(x) \nabla u(x)\right) \cdot \nabla \eta_{k}(x) d x \\
& -\int_{A_{K}} \nabla \beta_{k}(x) \cdot \nabla u(x) \eta_{k}(x) d x=H\left(u, A_{k}\right) .
\end{aligned}
$$

where in the last equality we have used again Lemma 3.1.

Therefore (4.5) implies that $\mathcal{G}$, being the supremum of the sum of supremum of lower continuous functionals is lower semicontinuous itself. Then since $g$ is convex it is not difficult to see that $\mathcal{F}$ is lower semicontinuous too.

\section{RELAXATION}

This section is devoted to the relaxation result. This result will be attained, once we will have proven the lower bound $\bar{F} \geq \mathcal{F}$ and the upper bound $\bar{F} \leq \mathcal{F}$. The first one is a consequence of the lower semicontinuity result proved in Theorem 4.1. In order to achieve upper bound we will strengthen the assumptions on the integrand $f$, by requiring uniform lower semicontinuity condition with respect to $x$.

5.1. Integral representation formula. We will assume that the integrands $f: \Omega \times$ $\mathbb{R} \rightarrow[0,+\infty)$ and $g: \mathbb{R}^{N} \rightarrow[0,+\infty)$ satisfy the following assumptions:

$$
f(x, t) \leq C_{1}(1+|t|) \quad \forall(x, t) \in \Omega \times \mathbb{R},
$$

where $0<C_{1}<+\infty$ is a constant;

$$
g(\xi) \leq C_{2}\left(1+|\xi|^{p}\right) \quad \forall \xi \in \mathbb{R}^{N},
$$

where $0<C_{2}<+\infty$ is a constant. Moreover from assumption (5.1) it follows that

$$
f^{\infty}(x, t) \leq C_{1}|t| \quad \forall(x, t) \in \Omega \times \mathbb{R} .
$$

We also assume that for all $\epsilon>0$ there exists $\delta>0$ such that for all $x \in \Omega$

$$
f(x, t) \leq f(y, t)+\epsilon(1+f(y, t)) \quad \forall(y, t) \in \Omega \times \mathbb{R} \text { such that }|x-y| \leq \delta
$$


which implies that for all $\epsilon>0$ there exists $\delta>0$ such that for all $x \in \Omega$

$$
f^{\infty}(x, t) \leq f^{\infty}(y, t)+\epsilon f^{\infty}(y, t) \quad \forall(y, t) \in \Omega \times \mathbb{R} \text { such that }|x-y| \leq \delta .
$$

Remark 5.1. Let us note that assumption (5.4) holds whenever the integrand $f$ is coercive with respect to the variable t for every fixed $x$ and satisfies the following stronger condition:

for all $\epsilon>0$ there exists $\delta>0$ such that for all $x \in \Omega$

$$
f(x, t)-f(y, t)<\epsilon|t| \quad \forall(y, t) \in \Omega \times \mathbb{R} \text { such that }|x-y| \leq \delta .
$$

This is the case, for instance, for $f(x, t)=|x t|$, if $0 \notin \Omega$.

Theorem 5.1. Let $f: \Omega \times \mathbb{R} \rightarrow[0,+\infty)$ be a Borel lower semicontinuous function convex in the second variable satisfying (4.1). Assume that (5.1),(5.2) and (5.4) hold. Then we have

$$
\mathcal{F}\left(u, \Omega^{\prime}\right)=\bar{F}\left(u, \Omega^{\prime}\right) \quad \forall u \in \Delta \mathcal{M}_{\mathrm{loc}}^{p}(\Omega) \quad \forall \Omega^{\prime} \subset \subset \Omega
$$

Proof. Step one: We start by proving the lower bound $\mathcal{F} \leq \bar{F}$.

Since $\bar{F}$ is the greatest lower semicontinuous functional not greater than $F, \mathcal{F} \leq F$, and by Theorem $4.1, \mathcal{F}$ is $W_{\text {loc }}^{1, p}$-weak lower semicontinuous, it follows that $\mathcal{F}\left(u, \Omega^{\prime}\right) \leq$ $\bar{F}\left(u, \Omega^{\prime}\right)$ for all $u \in \Delta \mathcal{M}_{\mathrm{loc}}^{p}(\Omega)$ and for all $\Omega^{\prime} \subset \subset \Omega$.

Step two: We now prove the upper bound $\mathcal{F} \geq \bar{F}$.

By taking into account assumption (5.4) we can apply Theorem 2.3 to the function $f$. So we have for all $(x, t) \in \Omega \times \mathbb{R}$

$$
f(x, t)=\sup _{k} a_{k}(x) \psi_{k}(t), \quad f^{\infty}(x, t)=\sup _{k} a_{k}(x) \psi_{k}^{\infty}(t),
$$

where $\psi_{k}$ are convex functions. Therefore it follows that there exist $\left\{\alpha_{k}^{l}\right\},\left\{\beta_{k}^{l}\right\} \subset \mathbb{R}$ such that for all $(x, t) \in \Omega \times \mathbb{R}$

$$
f(x, t)=\sup _{k} a_{k}(x)\left(\sup _{l}\left(\alpha_{k}^{l}+\beta_{k}^{l} t\right)\right) \quad f^{\infty}(x, t)=\sup _{k} a_{k}(x)\left(\sup _{l} \beta_{k}^{l} t\right) .
$$

Let now $u \in \Delta \mathcal{M}_{\text {loc }}^{p}(\Omega)$ and $\Omega_{h}=\left\{x \in \Omega\right.$ such that $\left.\operatorname{dist}(x, \partial \Omega)>\frac{1}{h}\right\}$. Let $\left\{u_{h}\right\} \subset$ $C^{\infty}\left(\Omega_{h}\right)$ defined as $u_{h}=u * \rho_{h}$ where $\rho_{h}$ a standard mollifying sequence, with $\operatorname{supp} \rho_{h}=$ $\left\{x \in \Omega ;|x| \leq \frac{1}{h}\right\}$. For $x \in \Omega_{h}$ we have that the support of $\rho_{h}(x-\cdot)$ is contained in $\Omega$. 
Then for every $x \in \Omega_{h}$ we have

$$
\begin{aligned}
& a_{k}(x)\left(\alpha_{l}^{k}+\beta_{k}^{l} \Delta u_{h}(x)\right)=a_{k}(x)\left(\int_{\Omega} \rho_{h}(x-y)\left(\alpha_{k}^{l}+\beta_{k}^{l} \Delta u(y)\right) d y\right. \\
+ & \left.\int_{\Omega} \rho_{h}(x-y) \beta_{k}^{l} \frac{d(\Delta u)^{s}}{\left|d(\Delta u)^{s}\right|}(y) d\left|(\Delta u)^{s}\right|\right) .
\end{aligned}
$$

By taking into account (5.6) and (5.7) we get:

$$
\begin{aligned}
& a_{k}(x)\left(\alpha_{l}^{k}+\beta_{k}^{l} \Delta u_{h}(x)\right) \leq \int_{\Omega} \rho_{h}(x-y) f(x, \Delta u(y)) d y \\
+ & \int_{\Omega} \rho_{h}(x-y) f^{\infty}\left(x, \frac{d(\Delta u)^{s}}{\left|d(\Delta u)^{s}\right|}(y)\right) d\left|(\Delta u)^{s}\right| .
\end{aligned}
$$

Let $\epsilon>0$ and $\delta$ be consequently given by assumption (5.4). Then for $h$ large enough, for $x \in \Omega_{h}$ and $y \in \Omega$ such that $|x-y| \leq \frac{1}{h}$, we have that $|x-y| \leq \frac{1}{h} \leq \delta$. Hence from (5.4), (5.5) it follows that for all $\epsilon>0$

$$
\begin{aligned}
& a_{k}(x)\left(\alpha_{l}^{k}+\beta_{k}^{l} \Delta u_{h}(x)\right) \leq \int_{\Omega} \rho_{h}(x-y) f(y, \Delta u(y)) d y \\
&+ \int_{\Omega} \rho_{h}(x-y) f^{\infty}\left(y, \frac{d(\Delta u)^{s}}{\left|d(\Delta u)^{s}\right|}(y)\right) d\left|(\Delta u)^{s}\right|+\epsilon \int_{\Omega} \rho_{h}(x-y)(1+f(y, \Delta u(y)) d y \\
&(5.8)+\epsilon \int_{\Omega} \rho_{h}(x-y) f^{\infty}\left(y, \frac{d(\Delta u)^{s}}{\left|d(\Delta u)^{s}\right|}(y)\right) d\left|(\Delta u)^{s}\right| .
\end{aligned}
$$

Let now $\Omega^{\prime} \subset \subset \Omega$. Since $\Omega_{h}$ is a sequence of open sets invading $\Omega$, we have for $h$ large enough $\Omega^{\prime} \subset \Omega_{h}$. Then by taking the supremum firstly over $l$ and over $k$ on the left hand side of (5.8), performing an integration over $\Omega^{\prime}$, with respect to the variable $x$, and by taking into account the growth conditions (5.1) and (5.3) we get for $h$ large enough

$$
\begin{aligned}
& \int_{\Omega^{\prime}} f\left(x, \Delta u_{h}(x)\right) d x \leq \int_{\Omega^{\prime}} d x \int_{\Omega} \rho_{h}(x-y) f(y, \Delta u(y)) d y \\
+ & \int_{\Omega^{\prime}} d x \int_{\Omega} \rho_{h}(x-y) f^{\infty}\left(y, \frac{d(\Delta u)^{s}}{\left|d(\Delta u)^{s}\right|}(y)\right) d\left|(\Delta u)^{s}\right| \\
+ & \epsilon\left(\int_{\Omega^{\prime}} d x \int_{\Omega} \rho_{h}(x-y) d y+\int_{\Omega^{\prime}} d x \int_{\Omega} \rho_{h}(x-y) d|\operatorname{Div} \nabla u|\right) \\
\leq & \int_{\Omega^{\prime}} d x \int_{\Omega} \rho_{h}(x-y) f(y, \Delta u(y)) d y+\int_{\Omega^{\prime}} d x \int_{\Omega} \rho_{h}(x-y) f^{\infty}\left(y, \frac{d(\Delta u)^{s}}{\left|d(\Delta u)^{s}\right|}(y)\right) d\left|(\Delta u)^{s}\right| \\
+ & \epsilon \mathcal{L}^{n}(\Omega) C_{1}\left(1+|\operatorname{Div} \nabla u|\left(\Omega^{\prime}\right)\right) .
\end{aligned}
$$

Finally by applying Fubini's Theorem and taking into account that $\epsilon$ is arbitrary we conclude

$$
\int_{\Omega^{\prime}} f\left(x, \Delta u_{h}\right) d x \leq \int_{\Omega^{\prime}} f(x, \Delta u) d x+\int_{\Omega^{\prime}} f^{\infty}\left(x, \frac{d(\Delta u)^{s}}{d\left|(\Delta u)^{s}\right|}\right) d\left|(\Delta u)^{s}\right|,
$$

where we have used that $\int_{\Omega} \rho_{h}(x-y) d x \leq \int_{\mathbb{R}^{N}} \rho_{h}(x-y) d x=1$. 
As in the proof of Theorem 3.1,2 in [15], we also get that

$$
\int_{\Omega^{\prime}} g\left(\nabla u_{h}\right) d x \leq \int_{\Omega^{\prime}} g(\nabla u) d x
$$

Then (5.9) and (5.10) imply

$$
\liminf _{h \rightarrow+\infty} \int_{\Omega^{\prime}} f\left(x, \Delta u_{h}\right) d x+\int_{\Omega^{\prime}} g\left(\nabla u_{h}\right) d x \leq \mathcal{F}\left(u, \Omega^{\prime}\right) .
$$

Therefore since $\left\{u_{h}\right\}_{h} \subset C^{2}\left(\Omega^{\prime}\right)$ and $u_{h}$ goes to $u$ with respect to the $W_{\text {loc }}^{1, p}$-convergence, from (5.11) it follows that

$$
\begin{aligned}
\bar{F}\left(u, \Omega^{\prime}\right) & =\inf _{u_{h} \subset C^{2}\left(\Omega^{\prime}\right)}\left\{\liminf _{h \rightarrow+\infty} F\left(u_{h}, \Omega^{\prime}\right) \quad u_{h} \rightarrow u\right\} \\
& \leq \liminf _{h \rightarrow+\infty} F\left(u_{h}, \Omega^{\prime}\right) \leq \mathcal{F}\left(u, \Omega^{\prime}\right) .
\end{aligned}
$$

This, being $\Omega^{\prime}$ arbitrary, concludes the step two and the whole proof. 
Acknowledgments: We warmly thank anonymous referee for its careful reading of the paper and numerous remarks. Its advices have permitted to significantly improve our work.

\section{REFERENCES}

[1] G.Anzellotti. Pairings between measures and bounded functions and compensated compactness. Ann. Mat. Pura Appl. 135 (1983), 293-318.

[2] G.Aubert, D.Graziani, L.Blanc-Féraud Analysis of a new variational model to restore point-like and curve-like singularities in imaging. To appear on Applied Mathematics and Optimization.

[3] G.Aubert, D.Graziani. Variational approximation of detecting point-like target problems. COCV: Esaim Control Optimisation and Calculus of Variations 4 (2011), pp. 909-930.

[4] A.Braides. $\Gamma$-convergence for beginners. Oxford University Press, New york (2000).

[5] G.Dal Maso. Introduction to $\Gamma$-convergence. Birkhäuser, Boston(1993).

[6] G.Q.Chen, H.Frid Divergence-measure fields and conservation laws. Arch. Rational Mech. Anal. 147 (1999), 35-51.

[7] G.Q.Chen, H.Frid. On the theory of divergence-measure fields and its applications. Bol. Soc. Bras. Math. 32 (2001), 1-33.

[8] G.Dal Maso, F.Murat, L.Orsina, A.Prignet. Renormalized solutions of elliptic equations with general measure data. Ann. Scuola Norm. Sup. Pisa Cl. Sci, 28 (1999), 741-808.

[9] E.De Giorgi. Semicontinuity theorems in the calculus of variations ; notes written by U. Mosco, G. Troianiello, G. Vergara / Napoli : Accademia Pontaniana , 2008

[10] E.De Giorgi, G.Buttazzo,G.Dal Maso. On the lower semicontinuity of certain integral functionals. Atti Accad. Naz. Lincei Rend. Cl. Sci. Fis. Mat. Natur. 74 (1983), 274-282.

[11] L.C.Evans, R.F. Gariepy. Measure Theory and Fine Properties of Functions. CRC Press (1992).

[12] N.Fusco, M.Gori, F.Maggi. A remark on Serrin's Theorem. Nonlinear Differential Equations Appl. 13 (2006), 425-433.

[13] D.Graziani On the $L^{1}$-lower semicontinuity and relaxation in $B V\left(\Omega ; \mathbb{R}^{M}\right)$ for integral functionals with discontinuous integrand. Phd Thesis (2006) available on cvgmt.sns.it server

[14] D.Graziani, L.Blanc-Féraud, G.Aubert. A formal $\Gamma$-convergence approach for the detection of points in 2-D images. Siam Journal of Imaging Science 3 (2010), 578-594.

[15] C.Goffmann, J.Serrin. Sublinear functions of measures and variation integrals. Duke Math J. 31 (1964), 159-178.

[16] F.Maggi On Serrin's lower semicontinuty theorem. Phd Thesis (2007) available on cvgmt.sns.it server.

[17] W.Ziemer. Weakly Differentiable Functions. Springer-Verlag, New york (1989). 